\title{
Study of Salmonella Isolates and their Antimicrobial Susceptibility Pattern in a Tertiary Care Hospital of NE-India
}

\author{
Anjan Sarma* and Dipa Barkataki \\ Department of Microbiology, Gauhati Medical College and Hospital, \\ Guwahati, Kamrup Assam, India \\ *Corresponding author
}

\begin{tabular}{|l|}
\hline K e y w o r d s \\
Blood culture, \\
$\begin{array}{l}\text { Salmonella, } \\
\text { Antimicrobial } \\
\text { sensitivity }\end{array}$ \\
\hline Article Info \\
\hline $\begin{array}{l}\text { Accepted: } \\
\text { 10 June } 2019 \\
\text { Available Online: } \\
\text { 10 July } 2019\end{array}$ \\
\hline
\end{tabular}

\section{A B S T R A C T}

Enteric fever is a global health problem and is also endemic in India. The etiologic agents of enteric fever i.e. S. typhi and S. paratyphi occurs mostly through food-borne or waterborne transmission. Worldwide, however, there are an estimated 22 million cases of enteric fever annually, with 600,000 deaths and an estimate of the annual typhoid incidence rate of 493.5 per 100,000 person years was reported from India in 2008. The laboratory diagnosis of enteric fever is very important mainly in this post-antibiotic era. Isolation of Salmonella by blood culture is proof that the patient has a salmonella septicemia and still is the definitive diagnosis for a patient. Antibiotic therapy is the only effective treatment for enteric fever. Currently, fluoroquinolones and third generation cephalosporins are drugs of choice for treatment of typhoid fever though recent reports of decreased susceptibility to these agents and emergence of resistance to chloramphenicol, ampicillin and cotrimoxazole have led to the prospect of reemergence of untreatable typhoid fever and an increasing global burden. A total of 110 nos. of blood samples were collected from patients (cases) clinically suspected of Enteric fever for a duration of one year from August'2016-July' 2017. Salmonella isolation was done by conventional methods of culture. Species identification was done by KBO11. Confirmed Salmonella isolates were tested by serotyping and antimicrobial susceptibility of isolated Salmonella spp were tested by modified Kirby-Bauer disc diffusion method as per the recommendation of Clinical and Laboratory Standard Institute (CLSI). Out of 110 blood culture samples, Salmonella spp were isolated and prevalence was $18.18 \%$ (20 cases), out of which S.typhi (18 cases-90 \%) was most commonly isolated organism followed by S. paratyphi (2cases $-10 \%$ ). $70 \%$ of the culture positive cases were males (14) and $30 \%$ were females (6), with a male and female ratio $2.33: 1$. The majority of the blood culture confirmed cases $(55 \%)$ belong to the age group of 21-40 years. Though enteric fever cases occurred in all months throughout the year, maximum cases occurred during the rainy season from April-July followed by August-September. All 18 S.Typhi isolates showed sensitivity to Ceftriaxone (100\%), Azithromycin (100\%) whereas sensitivity to Cotrimoxazole $(94.44 \%)$ and Chloramphenicol $(88.88 \%)$ was also high. $100 \%$ sensitivity was observed to third-generation cephalosporins. Least sensitivity was observed to Nalidixic acid. Though nowadays, rapid diagnostic procedures is being used to "suspect" enteric fever it is prudent to follow them up with blood culture confirmation. In-vitro antibiotic sensitivity should be routinely carried out in all patients of enteric fever for prompt and effective management which will minimise the indiscriminate use of antibiotics. 


\section{Introduction}

Enteric fever is a global health problem and is also endemic in India. It is a systemic disease characterized by fever and abdominal pain and caused by dissemination of $S$. typhi or $S$. paratyphi. The disease was initially called typhoid fever because of its clinical similarity to typhus. However, in the early 1800s, typhoid fever was clearly defined pathologically as a unique illness on the basis of its association with enlarged Peyer's patches and mesenteric lymph nodes. ${ }^{(1)}$

The etiologic agents of enteric fever i.e. S. typhi and $S$. paratyphi serotypes $\mathrm{A}, \mathrm{B}$, and $\mathrm{C}$-have no known hosts other than humans. Most commonly, food-borne or waterborne transmission results from fecal contamination by ill or asymptomatic chronic carriers. Health care workers occasionally acquire enteric fever after exposure to infected patients or during processing of clinical specimens and cultures. ${ }^{(1)}$

With improvements in food handling and water/sewage treatment, enteric fever has become rare in developed nations. Worldwide, however, there are an estimated 22 million cases of enteric fever annually, with 600,000 deaths. ${ }^{(2)}$

The incidence is highest $(>100$ cases per 100,000 population per year) in south central and Southeast Asia; medium (10-100 cases per 100,000) in the rest of Asia, Africa, Latin America, and Oceania (excluding Australia and New Zealand); and low in other parts of the world. (1) An estimate of the annual typhoid incidence rate of 493.5 per 100,000 person years was reported from India in 2008 in the Bulletin of the World Health Organization. $^{(3)}$

A high incidence of enteric fever correlates with poor sanitation and lack of access to clean drinking water. ${ }^{(1)}$ The highest incidence occurs in the regions where water supply serving large populations is contaminated with faeces. ${ }^{(4)}$

A recent outbreak of typhoid fever occurred in Jorhat town of Assam, India in the January'2014 which occurred due to S. typhi $(46.15 \%)$ contaminating the water supply ${ }^{(6)}$.

The laboratory diagnosis of enteric fever is very important mainly because in postantibiotic era most of the patients are treated empirically by the local medical practitioners and when the fever does not subside, these cases are labeled as pyrexia of unknown origin (PUO) and investigated for various causes of PUO including enteric fever. At this stage the typical signs and symptoms are hardly observed. ${ }^{(5)}$

The presence of $S$. typhi or $S$. paratyphi is detected either by culture of the organism or by the demonstration of specific antibodies or antigen in the serum. (3) Isolation of a Salmonella by blood culture is proof that the patient has a Salmonella septicemia. Isolation from the feces is of less certain significance. Since Salmonella may be present in the faeces of carriers, it does not amount to proof of a causal role. ${ }^{(7)}$

There is an urgent need for the rational design and evaluation of effective and appropriate diagnostic tools for enteric fever which must include the emerging threat of S. Paratyphi. ${ }^{(8)}$

Antibiotic therapy is the only effective treatment for enteric fever. Although effective treatment with chloramphenicol was introduced in 1948, the emergence of resistance to chloramphenicol, ampicillin and cotrimoxazole has been of concern. Currently, fluoroquinolones and third generation cephalosporins are drugs of choice for treatment of typhoid fever. However, recent 
reports of decreased susceptibility to these agents have led to the prospect of reemergence of untreatable typhoid fever and an increasing global burden. ${ }^{(9)}$

Keeping in view all the above, the present study was undertaken at $\mathrm{GMCH}$, Assam to study the prevalence of Salmonella species in clinically suspected cases of Enteric fever and to study the antibiotic susceptibility pattern of the isolates.

\section{Materials and Methods}

A total of 110 nos. of blood samples were collected from patients (cases) clinically suspected of Enteric fever for a duration of one year from August'2016-July' 2017.

The study was commenced with ethical approval and clearance certificate from the IEC, GMCH.

Salmonella isolation was done by conventional methods of culture. Species identification was done by KBO11 (HIMEDIA). Confirmed Salmonella isolates were tested by slide agglutination test using commercially prepared anti-sera using $\mathrm{O}$ typing sera for group identification and $\mathrm{H}$ typing sera for type identification. Antimicrobial susceptibility of isolated Salmonella spp were tested by modified Kirby-Bauer disc diffusion method as per the recommendation of Clinical and Laboratory Standard Institute (CLSI). E. coli 25922 ATCC were used as quality control strains.

\section{Inclusion criteria}

1) Patients attending outpatient department and in-patients of various departments clinically suspected to be suffering from enteric fever were included in the study.

2) Patients suspected with having enteric (typhoid) fever may have the following signs and symptoms:

Gradual onset fever with headache, malaise, anorexia, a coated tongue and abdominal discomfort with either constipation or diarrhea.

Step-ladder pyrexia, with relative bradycardia and toxemia.

A soft, palpable spleen with hepatomegaly.

Rose-spots that fade on pressure (mainly appear during 2nd or 3rd week of enteric fever).

3) Individuals of both sexes representing all ages.

\section{Exclusion criteria}

1) Patients having no signs and symptoms of Enteric (typhoid) fever.

2) Fever with any obvious focus for other infection such as Urinary tract infection, Blood septicaemia, Fungal infection, Otitis media etc.

3) Patients with prior antibiotic administration and $\mathrm{TAB}$ vaccination.

\section{Results and Discussion}

Out of 110 cases, 75 cases were male and 35 were female. Majority of the cases $(45.45 \%)$ belong to the age group of 21-40 years with overall male-female ratio 2.14: 1. Majority of the male cases $46.67 \%(35 / 75)$ and female cases $42.85 \%$ (15/35) belonged to age group of 21-40years.

Out of 110 cases, $18.18 \%$ (20 cases) were confirmed by blood culture. Similar observation was seen in study done by Patel et al., (2013) ${ }^{(10)}$ and Bhattacharya et al., $(2017)^{(11)}$. Relative low rate of isolation from blood culture has been attributed to delay in visiting the physician for diagnosis, wide 
spread antibiotic use and the difficulties of obtaining large enough blood volumes. 70\% of the culture positive cases were males (14) and $30 \%$ were females (6), with a male and female ratio 2.33:1 which correlates with the studies of Sood and Taneja ${ }^{(12)}$ and Jog et al.,
$(2008)^{(13)}$. This might be due to our cultural background where male is more likely to report to hospital, at the same time more likely to contract infection due to more outdoor activities (Table 1-3).

Table.1 Age distribution of cases

\begin{tabular}{|ccccccc|}
\hline Age (in yrs) & Male & Percentage & Female & Percentage & Total & Percentage \\
\hline $\mathbf{0 - 2 0}$ & $\mathbf{5}$ & $\mathbf{6 . 6 7} \%$ & $\mathbf{2}$ & $\mathbf{5 . 7 1} \%$ & $\mathbf{7}$ & $\mathbf{6 . 3 6} \%$ \\
\hline $\mathbf{2 1 - 4 0}$ & $\mathbf{3 5}$ & $\mathbf{4 6 . 6 7} \%$ & $\mathbf{1 5}$ & $\mathbf{4 2 . 8 5} \%$ & $\mathbf{5 0}$ & $\mathbf{4 5 . 4 5 \%}$ \\
\hline $41-60$ & $\mathbf{2 0}$ & $\mathbf{2 6 . 6 7} \%$ & $\mathbf{1 0}$ & $\mathbf{2 8 . 5 7} \%$ & $\mathbf{3 0}$ & $\mathbf{2 7 . 2 8} \%$ \\
\hline$>\mathbf{6 0}$ & $\mathbf{1 5}$ & $\mathbf{2 0} \%$ & $\mathbf{8}$ & $\mathbf{2 2 . 8 5} \%$ & $\mathbf{2 3}$ & $\mathbf{2 0 . 9} \%$ \\
\hline Total & $\mathbf{7 5 / 1 1 0}$ & $\mathbf{6 8 . 1 8 \%}$ & $\mathbf{3 5} / 110$ & $\mathbf{3 1 . 8 2} \%$ & $\mathbf{1 1 0}$ & \\
\hline
\end{tabular}

Table.2 Age and sex distribution of culture positive cases

\begin{tabular}{|c|c|c|c|c|}
\hline \multirow{2}{*}{ Age (inyears) } & \multicolumn{3}{|c|}{ Positive cases confirmed by Culture method } & \\
\cline { 2 - 5 } & Male & Female & Total & Percentages \\
\hline $0-20$ & 2 & 0 & 2 & 10 \\
\hline $21-40$ & 8 & 3 & 11 & $55 \%$ \\
\hline $41-60$ & 2 & 1 & 3 & $15 \%$ \\
\hline$>60$ & 2 & 2 & 4 & $20 \%$ \\
\hline Total & $\mathbf{1 4}$ & $\mathbf{6}$ & $\mathbf{2 0}$ & \\
\hline Percentage & $\mathbf{7 0 \%}$ & $\mathbf{3 0 \%}$ & & \\
\hline
\end{tabular}

Table.3 AST of total 20 isolated Salmonella group of organisms

\begin{tabular}{|c|c|c|c|c|}
\hline Antibiotic Discs & $\begin{array}{c}\text { Abbreviati } \\
\text { on }\end{array}$ & $\begin{array}{c}\text { Sensitive } \\
(\%)\end{array}$ & $\begin{array}{c}\text { Intermediate } \\
(\%)\end{array}$ & $\begin{array}{c}\text { Resistant } \\
(\%)\end{array}$ \\
\hline Ampicillin & AMP & $75 \%(15)$ & - & $25 \%(5)$ \\
\hline $\begin{array}{c}\text { Cotrimoxazole } \\
\text { (sulfamethoxazole+Tri } \\
\text { methoprim) }\end{array}$ & COT & $95 \%(19)$ & - & $5 \%(1)$ \\
\hline Ceftriaxone & CTR & $100 \%(20)$ & - & - \\
\hline Ciprofloxacin & CIP & $100 \%(20)$ & - & - \\
\hline Azithromycin & AZM & $90 \%(18)$ & - & $10 \%(2)$ \\
\hline Chloramphenicol & C & $85 \%(17)$ & - & $15 \%(3)$ \\
\hline Nalidixic acid & NA & $60 \%(12)$ & - & $40 \%(8)$ \\
\hline
\end{tabular}


Graph.1 Age distribution of total cases

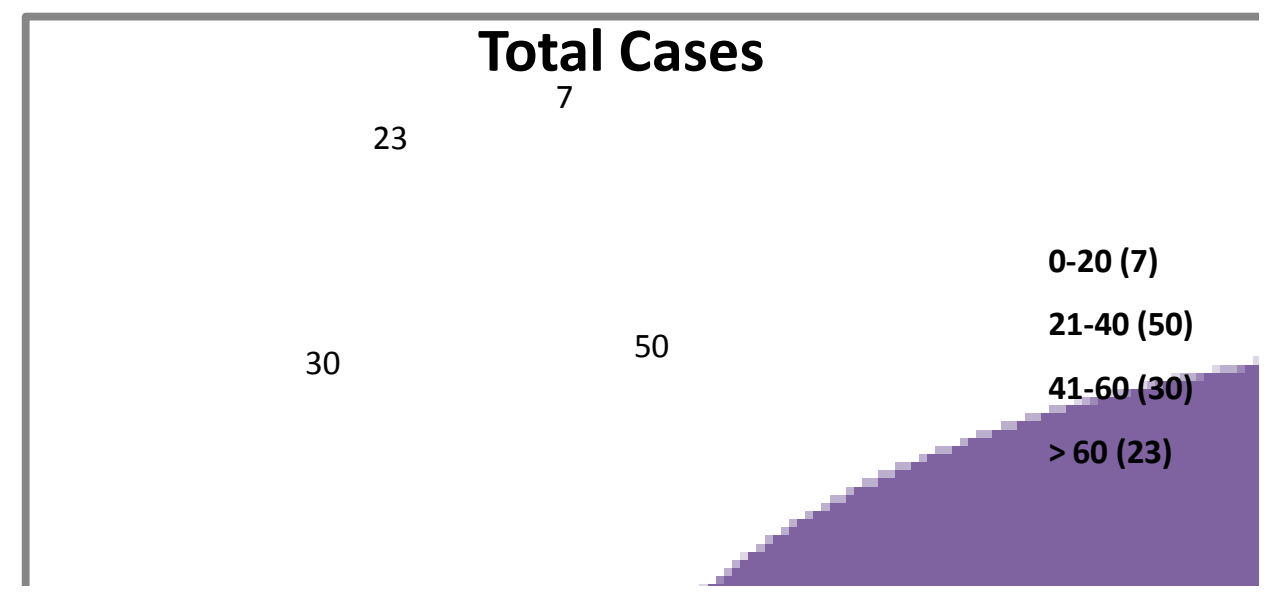

Graph.2 Age and sex distribution of culture positive cases

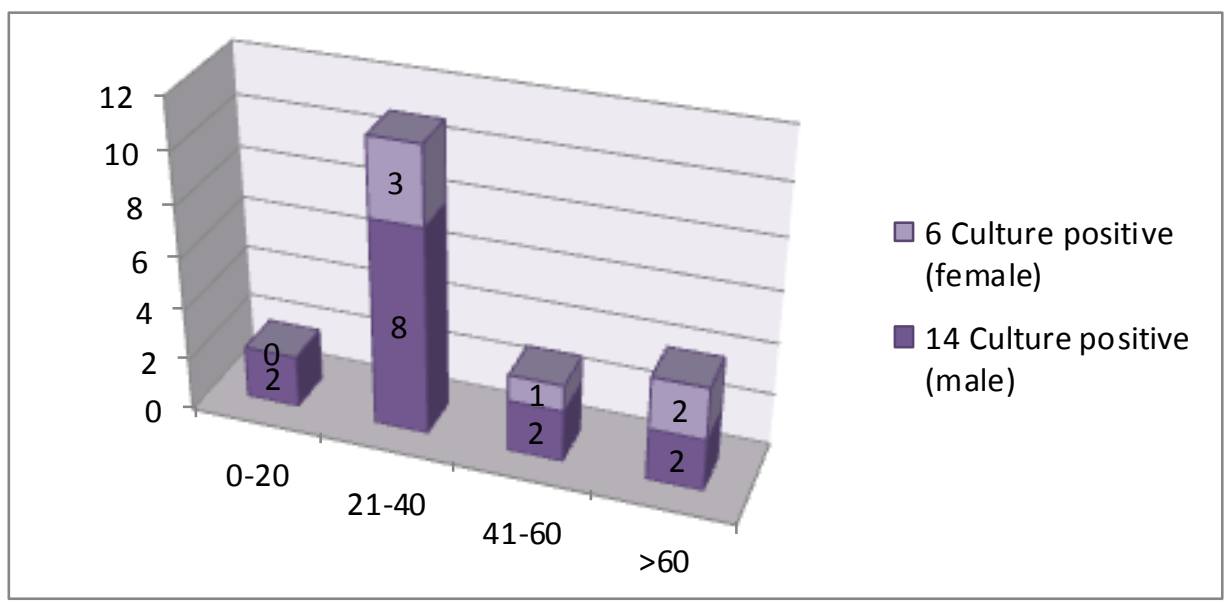

Graph.3 Seasonal variation of cases

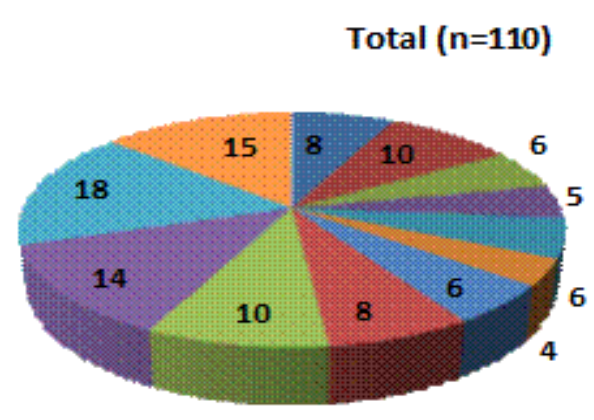

$$
\begin{aligned}
& \text { nugust } \\
& \text { 四 Septembe } \\
& r \\
& \text { October } \\
& \text { November } \\
& \text { 밈 December } \\
& \text { 치 } \\
& \text { eㅡㄹ }
\end{aligned}
$$


Graph.4 Species distribution of 20 Salmonella isolates

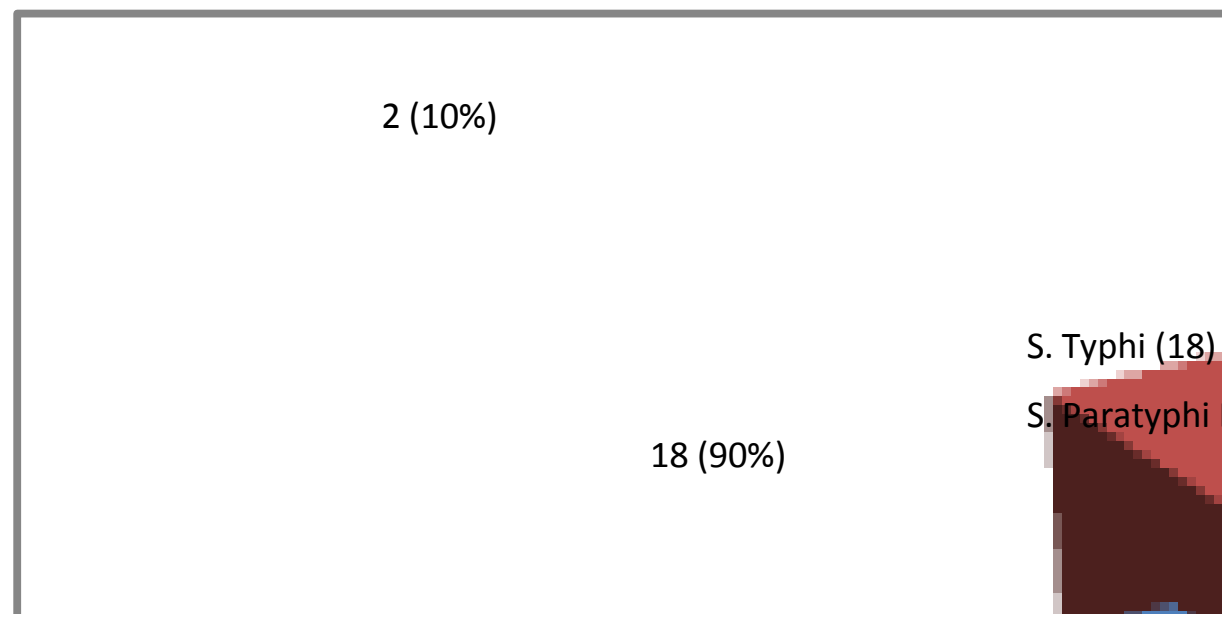

The majority of the blood culture confirmed cases $(55 \%)$ belong to the age group of 21-40 years which correlates with the study done by of J Sharma et al., (2013) ${ }^{(14)}$. The possible causes for enteric fever being common in age group 21-40 yrs include their mobility, consumption of unhygienic food outside home and drinking of contaminated or unfiltered water in colleges and offices or at their jobs. Majority of Blood culture positive $(75 \%)$ were detected in the $1^{\text {st }}$ week of illness. In the present study, enteric fever cases occurred in all months throughout the year though maximum cases occurred during the rainy season from April-July followed by August-September. Similar observation seen in studies done by Singhal et al., (2013) $)^{(15)}$ and J Sharma et al., (2013) ${ }^{(14)}$. This occurs probably due to the higher chances of water contamination.

In this present study, out of positive blood cultures, S. typhi (18 cases-90 \%) was most commonly isolated organism followed by $S$. paratyphi (2 cases $-10 \%)$. Similar observations are seen in study done by Manchanda et al., (2006) ${ }^{(16)}$, Gupta et al., $(1985)^{(17)}$ and Prasad et al., $(2015)^{(18)}$
It is observed in our study that the 20 Salmonella isolates showed sensitivity to Ceftriaxone (100\%) and Ciprofloxacin (100 $\%)$, followed by Azithromycin (90 \%), Cotrimoxazole (90\%), Chloramphenicol(85 $\%)$, Ampicillin (75\%) and Nalidixic acid $(60 \%)$. Similar observation was seen in study done by Ashwini et al., (2013) ${ }^{(19)}$ and Behl et al., $(2017)^{(20)}$. Patel et al., $(2013)^{(10)}$ which reported $100 \%$ sensitivity to Azithromycin and Ofloxacin. S Dahiya et al., (2017) ${ }^{(22)}$ reported $100 \%$ sensitivity to third-generation cephalosporins which correlates with the present study.

All $18 S$. typhi isolates showed sensitivity to Ceftriaxone (100\%), Ciprofloxacin (100\%), Azithromycin (100 \%) whereas some sensitivity to Cotrimoxazole (94.44\%), Chloramphenicol $(88.88 \%)$, Ampicillin $(83.33 \%)$ and Nalidixic acid (55.55 \%) were also observed.

The present study correlates with studies done by Patel et al., (2017) ${ }^{(23)}$ who reported 98\% S. typhi sensitivity to Chloramphenicol and least sensitivity to Nalidixic acid (23.4\%) and Kumar Y et al., (2011) ${ }^{(21)}$ which reported $S$. typhi isolates to be sensitive to 
Chloramphenicol (95.3\%) and Trimethoprim $(94.5 \%)$.

$100 \%$ of $S$. paratyphi B sensitive to Ciprofloxacin, Ceftriaxone and Cotrimoxazole. $50 \%$ of $S$. paratyphi were sensitive to Ampicillin, Chloramphenicol and Nalidixic acid. None of $S$. paratyphi were sensitive to Azithromycin.

In conclusion, after analyzing it was concluded that the clinical diagnosis of enteric fever is a true diagnostic challenge as it may simulate many fever causing infectious diseases. Definitive diagnosis is still the isolation of the causative organism from the patient. Though nowadays, rapid diagnostic procedures can be used to "suspect" enteric fever more strongly than on clinical grounds alone, it is prudent to follow them up with blood culture confirmation. In-vitro antibiotic sensitivity should be routinely carried out in all patients of enteric fever for prompt and effective management which will minimise the indiscriminate use of antibiotics, minimise occurrence of drug resistance and reduce the economic and morbidity burden in hospitals.

\section{References}

1. Harrison's Principle of Internal Medicine, 18/e: Chapter 153, PublisherMcGrawHill.

2. Ananthanarayan R. Textbook of microbiology. University Press (India); 2013.;288-301

3. Ochiai RL, Acosta CJ, DanovaroHolliday MC, Baiqing D, Bhattacharya SK, Agtini MD, et al. A study of typhoid fever in five Asian countries: disease burden and implications for controls. Bull World Health Organ 2008; 86: 260-8.

4. World Health Organization. Background document: The diagnosis, treatment and prevention of typhoid fever. Communicable disease surveillance and response. Department of Vaccines and Biologicals. Geneva: WHO; 2003. p4-13

5. Sarman Singh: Symposium: Typhoid Fever- Pathogenesis and Laboratory Journal, Indian Academy of Clinical Medicine. Vol. 2, No. 1 and 2, JanuaryJune 2001, 18-20

6. Roy JS, Saikia L, Medhi M, Tassa D. Epidemiological investigation of an outbreak of typhoid fever in Jorhat town of Assam, India. The Indian journal of medical research. 2016 Oct; 144(4):592.

7. Gold, D.C: Salmonella, Sera for Identification of Salmonellae; Mackie \& McCartney Practical Medical Microbiology. $14^{\text {th }}$ edition. Churchill Livingstone; 2006: 401

8. Wain J, Hosoglu S.: The laboratory diagnosis of enteric fever. The Journal of Infection in Developing Countries. 2008 Dec 1; 2(6): 421-425.

9. Kothari A, Pruthi A, Chugh TD. The burden of enteric fever. The Journal of Infection in Developing Countries. 2008 Aug 1; 2(04): 253-9.

10. Patel KK, Majumdar D, Patel S, Sujatha R, Singh DN, Patel KK. Emerging ciprofloxacin and multi drug resistant Salmonella species isolated from patients with enteric fever in Chhattisgarh. Journal of Evolution of Medical and Dental Sciences. 2013 Mar 18; 2(11): $1638-43$.

11. Bhattacharya P, Saha BK, Paul UK, Bandyopadhyay A. Blood Culture in Clinically Suspected Typhoid Fever. Int J Sci Stud. 2017; 4(11):1-4.

12. Sood SC, Taneja PN. Typhoid Fever. Clinical Picture and Diagnosis. Indian Journal of Child Health. 1961;10(2):6976

13. Jog S, Soman R, Singhal T, Rodrigues C, Mehta A, Dastur FD. Enteric fever in Mumbai-clinical profile, sensitivity patterns and response to antimicrobials. JAPI. 2008 Apr 16; 56: 237-40. 
14. Sharma J, Malakar M. Distribution of Typhoid fever in different rural and urban areas of Lakhmipur District of Assam. International journal of research and development of health. 2013;1(3):109-14.

15. Singhal L, Gupta PK, Kale P, Gautam V, Ray P. Trends in antimicrobial susceptibility of Salmonella Typhi from North India (2001-2012). Indian journal of medical microbiology. 2014 Apr 1; 32(2): 149.

16. Manchanda V, Bhalla P, Sethi M, Sharma VK. Treatment of enteric fever in children on the basis of current trends of antimicrobial susceptibility of Salmonella enterica serovar typhi and paratyphi A. Indian journal of medical microbiology. 2006 Apr 1; 24(2): 101.

17. Gupta SP, Gupta MS, Bhardwaj S, Chugh TD. Current clinical patterns of typhoid fever: a prospective study. The Journal of tropical medicine and hygiene. $1985 \mathrm{Dec}$; 88(6): 377-81.

18. Prasad KJ, Oberoi JK, Goel N, Wattal C. Comparative evaluation of two rapid Salmonella-IgM tests and blood culture in the diagnosis of enteric fever. Indian journal of medical microbiology. 2015 Apr 1;33(2):237.

19. Ashwini Choudhury, Gopalakrishnan R, Senthur NP, Ramasubramanian V, Ghafur KA, Thirunarayan MA. Antimicrobial susceptibility of Salmonella enterica serovars in a tertiary care hospital in southern India. The Indian journal of medical research. 2013 Apr; 137(4): 800.

20. Behl P, Gupta V, Sachdev A, Guglani V, Chander J. Patterns in antimicrobial susceptibility of Salmonellae isolated at a tertiary care hospital in northern India. The Indian Journal of Medical Research. 2017 Jan; 145(1): 124.

21. Kumar Y, Sharma A, Mani KR. Reemergence of susceptibility to conventionally used drugs among strains of Salmonella typhi in central west India. The Journal of Infection in Developing Countries. 2011 Mar 21; 5(03): 227-30.

22. Dahiya S, Sharma P, Kumari B, Pandey S, Malik R, Manral N, Veeraraghavan B, Pragasam AK, Ray P, Gautam V, Sistla S. Characterisation of antimicrobial resistance in Salmonellae during 20142015 from four centres across India: An ICMR antimicrobial resistance surveillance network report. Indian Journal of Medical Microbiology. 2017 Jan 1;35(1):61.

23. Patel SR, Bharti S, Pratap CB, Nath G. Drug Resistance Pattern in the Recent Isolates of Salmonella typhi with Special Reference to Cephalosporins and Azithromycin in the Gangetic Plain. Journal of clinical and diagnostic research: JCDR. 2017 Jun;11(6):DM01

\section{How to cite this article:}

Anjan Sarma and Dipa Barkataki. 2019. Study of Salmonella Isolates and their Antimicrobial Susceptibility Pattern in a Tertiary Care Hospital of NE-India. Int.J.Curr.Microbiol.App.Sci. 8(07): 939-946. doi: https://doi.org/10.20546/ijcmas.2019.807.113 